\title{
Lexical Event Ordering with an Edge-Factored Model
}

\author{
Omri Abend, Shay B. Cohen and Mark Steedman \\ School of Informatics, University of Edinburgh \\ Edinburgh EH8 9AB, United Kingdom \\ \{oabend, scohen, steedman\}@inf.ed.ac.uk
}

\begin{abstract}
Extensive lexical knowledge is necessary for temporal analysis and planning tasks. We address in this paper a lexical setting that allows for the straightforward incorporation of rich features and structural constraints. We explore a lexical event ordering task, namely determining the likely temporal order of events based solely on the identity of their predicates and arguments. We propose an "edgefactored" model for the task that decomposes over the edges of the event graph. We learn it using the structured perceptron. As lexical tasks require large amounts of text, we do not attempt manual annotation and instead use the textual order of events in a domain where this order is aligned with their temporal order, namely cooking recipes.
\end{abstract}

\section{Introduction}

Temporal relations between events are often implicit, and inferring them relies on lexical and world knowledge about the likely order of events. For instance, to execute the instruction "fry the onion," the hearer should probably obtain oil beforehand, even if not instructed so explicitly. Lexical knowledge about the likely order of events is therefore necessary for any semantic task that requires temporal reasoning or planning, such as classifying temporal relations (Mani et al., 2006; Lapata and Lascarides, 2006; Yoshikawa et al., 2009; D'Souza and Ng, 2013; Mirza and Tonelli, 2014, inter alia), textual entailment (Dagan et al., 2013) or temporal information extraction (Ling and Weld, 2010). Lexical temporal knowledge is further important for model- ing grammatical phenomena such as tense and aspect (Steedman, 2002).

In this paper we address the task of lexical event ordering, namely predicting the ordering of events based only on the identity of the words comprising their predicates and arguments. Concretely, the task is to predict the order of an unordered set of predicate-argument structures. Predicting the likely order of event types is a step towards more intricate planning and reasoning scenarios (see $\S 3$ ), and is useful in itself for tasks such as conceptto-text generation (Reiter et al., 2000), or in validating the correctness of instruction sets. A related idea can be found in modeling sentence coherence (Lapata, 2003; Barzilay and Lapata, 2008, inter alia), although here we focus on lexical relations between events, rather than coherence relations between complete sentences.

Compiling a resource of temporal tendencies between events can hardly be done manually, given the number and wealth of phenomena that have to be accounted for. Temporally annotated corpora, often annotated according to TimeML principles (Pustejovsky et al., 2003), are a useful resource for studying temporal relations. However, due to incurred costs, annotated corpora are too small for most lexical tasks. For instance, the TimeML annotated data used in the latest TempEval shared task contains only $100 \mathrm{~K}$ words or so (UzZaman et al., 2013).

Previous work that does not rely on manually annotated data has had some success in discovering temporal lexical relations between predicates (Chklovski and Pantel, 2004; Chambers and Jurafsky, 2008b; Talukdar et al., 2012). However, despite their appeal, these methods have mostly fo- 
cused on inducing simple event types, consisting of single words (e.g., "buy-own") or fixed expressions, and are hard to extend to include rich features (e.g., order-based and pattern-based features). Furthermore, measuring recall without annotated data is notoriously difficult, and evaluation is often precisionbased or extrinsic.

We take a graph-based structured prediction approach to the task, motivated by the flexibility it allows in incorporating various feature sets and constraints. We use an edge-factored model, which decomposes over the edges in the graph of events comprising the recipe $(\S 4)$. We estimate the model using the structured perceptron algorithm. We compare the structured perceptron approach to an approximate greedy baseline and to a locally normalized model reminiscent of common approaches for order learning, obtaining superior results $(\S 8)$. The learning algorithm is of potential use in other ordering tasks such as machine translation reordering (Tromble and Eisner, 2009).

We focus on domains in which the order of events in the text is aligned with their temporal order. By doing so we avoid the costly and error-prone manual annotation of temporal relations by using the textual order of recipes to approximate their temporal order. ${ }^{1}$ Specifically, we address the cooking recipes domain, which we motivate in $\S 2$.

In summary, the contribution of this paper is three-fold: (1) we explore the task of lexical event ordering and means for its evaluation; (2) we present an edge-factored model for the task, and show it can be used to predict the order of events well $(77.7 \%$ according to standard measures for ordering evaluation); (3) we present a method for extracting events and create a dataset of ordered events using recipes extracted from the web.

\section{Related Work}

Temporal semantics is receiving increasing attention in recent years. Lexical features are in frequent use and rely in most part on external resources which are either manually compiled or automatically induced. The line of work most closely related to ours focuses on inducing lexical relations between

\footnotetext{
${ }^{1}$ See Cassidy et al. (2014) for a discussion of inter-annotator agreement in TimeML-based schemes.
}

event types. Most work has been unsupervised, often using pattern-based approaches relying on manually crafted (Chklovski and Pantel, 2004) or induced patterns (Davidov et al., 2007), that correlate with temporal relations (e.g., temporal discourse connectives). Talukdar et al. (2012) uses the textual order of events in Wikipedia biographical articles to induce lexical information. We use both textual order and discourse connectives to define our feature set, and explore a setting which allows for the straightforward incorporation of additional features.

Chambers and Jurafsky (2008b; 2009) addressed the unsupervised induction of partially ordered event chains (or schema) in the news domain, centered around a common protagonist. One of their evaluation scenarios tackles a binary classification related to event ordering, and seeks to distinguish ordered sets of events from randomly permuted ones, yielding an accuracy of 75\%. Manshadi et al. (2008) used language models to learn event sequences and conducted a similar evaluation on weblogs with about $65 \%$ accuracy. The classification task we explore here is considerably more complex (see $\$ 8$ ).

The task of script knowledge induction has been frequently addressed in recent years. Balasubramanian et al. (2013) and Pichotta and Mooney (2014) extended Chambers and Jurafsky's model to include events that have multiple arguments. Jans et al. (2012) use skip-grams to capture event-event relations between not necessarily consecutive events.

Regneri et al. (2010) constructed a temporal lexical knowledge base through crowd-sourcing. Their approach is appealing as it greatly reduces the costs incurred by manual annotation and can potentially be used in conjunction with lexical information obtained from raw text. Modi and Titov (2014) jointly learns the stereotypical order of events and their distributional representation, in order to capture paraphrased instances of the same event type. Frermann et al. (2014) models the joint task of inducing event paraphrases and their order using a Bayesian framework. All latter three works evaluated their induced temporal ordering knowledge on a binary prediction of whether a temporal relation between a pair of (not necessarily related) events holds, and not on the prediction of a complete permutation given an unordered event set as in this work. Their evaluation was conducted on 30 event pairs manually an- 
notated through crowd-sourcing, where Modi and Titov (2014) further included an evaluation on a large set of pairs automatically extracted from the Gigaword corpus.

The appeal of the cooking domain for studying various semantic phenomena has been recognized by several studies in NLP and AI (Tasse and Smith, 2008; Bollini et al., 2013; Cimiano et al., 2013; Regneri et al., 2013; Malmaud et al., 2014). The domain is here motivated by several considerations. First, recipes mostly describe concrete actions, rather than abstract relations, which are less relevant to temporal ordering. Second, from a practical point of view, many recipes are available online in computerreadable format. Third, the restrictiveness of the cooking domain can also be seen as an advantage, as it can reveal major conceptual challenges raised by the task, without introducing additional confounds.

\section{Temporally Ordering Lexical Events}

We formalize our task as follows. Let $U$ be a set of event types, namely actions or states (represented as predicates) and objects which these actions operate on (represented as arguments to the predicates; mostly ingredients or kitchenware). Formally, each $e \in U$ is a tuple $\left\langle a, c_{1}, \ldots, c_{n}\right\rangle$ where $a$ is the main verb or predicate describing the event (such as "stir" or "mix") and $c_{1}, \ldots, c_{n}$ is a list of arguments that the predicate takes (e.g., "salt" or "spoon"). Two additional marked events, $s$ and $f$, correspond to "start" and "finish" events. A recipe is a sequence of events in $U$, starting at $s$ and ending at $f$.

Given a recipe $R=\left\langle e_{1}, \ldots, e_{m}\right\rangle$, we wish to predict the order of the events just from the (multi)set $\left\{e_{i}\right\}_{i=1}^{m}$. In this work we use the textual order of events to approximate their temporal order (see, e.g., Talukdar et al. (2012) for a similar assumption). The validity of this assumption for cooking recipes is supported in $\S 6$.

Figure 1 gives an example of a set of events extracted from our dataset for the dish "Apple Crisp Ala [sic] Brigitte." Lexical information places quite a few limitations on the order of this recipe. For instance, in most cases serving is carried out at the end while putting the ingredients in is done prior to baking them. However, lexical knowledge in itself is unlikely to predict the exact ordering of the events as given in the recipe (e.g., spreading butter might be done before or after baking).

One of the major obstacles in tackling planning problems in AI is the knowledge bottleneck. Lexical event ordering is therefore a step towards more ambitious goals in planning. For instance, temporal relations may be used to induce planning operators (Mourão et al., 2012), which can in turn be used to generate a plan (recipe) given a specified goal and an initial set of ingredients.

\section{Model, Inference and Learning}

In this section we describe the main learning components that compose our approach to event ordering.

\subsection{Edge-Factored Model for Event Ordering}

We hereby detail the linear model we use for ordering events. Let $S=\left\{e_{1}, \ldots, e_{m}\right\} \subseteq U$ be a set of events as mentioned in $\S 3$. Let $G(S)=$ $(S \cup\{s, f\}, E(S))$ be an almost-complete directed graph with $E(S)=(S \cup\{s\}) \times(S \cup\{f\}) \subseteq U \times U$. Every Hamiltonian path ${ }^{2}$ in $G(S)$ that starts in $s$ and ends in $f$ defines an ordering of the events in $S$. The edge $\left(e_{i}, e_{j}\right)$ in such a path denotes that $e_{i}$ is the event that comes before $e_{j}$.

The modeling problem is to score Hamiltonian paths in a given directed graph $G(S)$. Here, we use an edge-factored model. Let $\phi:(U \times U) \rightarrow \mathbb{R}^{d}$ be a feature function for pairs of events, represented as directed edges. In addition, let $\theta \in \mathbb{R}^{d}$ be a weight vector. We define the score of a Hamiltonian path $h=\left(h_{1}, \ldots, h_{m+1}\right)\left(h_{i} \in E(S)\right)$ as:

$$
\operatorname{score}(h \mid S)=\sum_{i=1}^{m+1} \theta^{\top} \phi\left(h_{i}\right)
$$

Given a weight vector $\theta$ and a set of events $S$, inference is carried out by computing the highest scoring Hamiltonian path in $G(S)$ :

$$
h^{*}=\arg \max _{h \in H(S)} \operatorname{score}(h \mid S)
$$

where $H(S)$ is the set of Hamiltonian paths in $G(S)$ that start with $s$ and end with $f$. The path $h^{*}$ is the best temporal ordering of the set of events $S$ according to the model in Eq. 1 with weight vector $\theta$.

\footnotetext{
${ }^{2} \mathrm{~A}$ path in a graph that visits all nodes exactly once.
} 
(a)

$$
\begin{aligned}
& e_{1}=\langle\text { butter }, \text { dish }\rangle \\
& e_{2}=\langle\text { put }, \text { apples }, \text { water }, . . . \\
&\quad \text { flour }, \text { cinnamon }, \text { it }\rangle \\
& e_{3}=\langle\text { mix }, \text { with spoon },\rangle \\
& e_{4}=\langle\text { spread }, \text { butter }, \text { salt }, . . . \\
&\quad \text { over mix }\rangle \\
& e_{5}=\langle\text { bake, } F\rangle \\
& e_{6}=\langle\text { serve, cream, cream }\rangle
\end{aligned}
$$

(b) Butter a deep baking dish, put apples, water, flour, sugar and cinnamon in it. Mix with spoon and spread butter and salt over the apple mix. Bake at 350 degrees F until the apples are tender and the crust brown, about 30 minutes. Serve with cream or whipped cream.

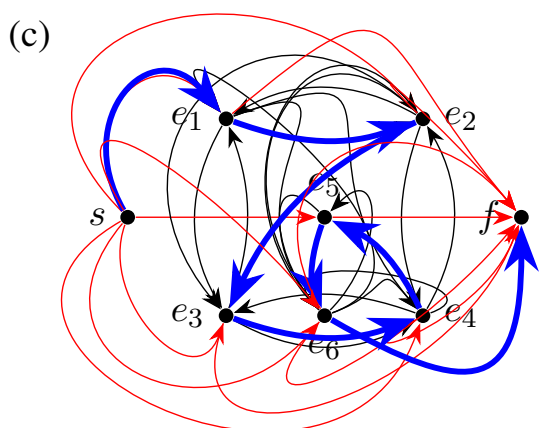

Figure 1: (a) The sequence of events representing the recipe for the dish "Apple Crisp Ala [sic] Brigitte." (b) The actual recipe for this dish. (c) A complete graph over the set of events with start and finish states. Each internal node in the graph is one of the events $e_{i}$ for $i \in\{1, \ldots, 6\}$. The path in blue bold denotes the correct Hamiltonian path describing the set of actions as ordered in the recipe. Red edges denote edges from the start state and to the end state. The edges, in practice, are weighted.

\subsection{Inference}

As mentioned above, inference with the edgefactored model requires solving the maximization problem in Eq. 2. This corresponds to finding a Hamiltonian path in a complete graph, which is generally an NP-hard problem. Reasonable approximations for this problem are also NP-hard. Still techniques are developed for specialized cases, due to the problem's importance in discrete optimization.

Despite its theoretical NP-hardness, this maximization problem can be represented as an Integer Linear Program (ILP), and then solved using generic techniques for ILP optimization. Due to the relatively short length of recipes (13.8 events on average in our corpus), the problem can be effectively solved in most cases.

The proposed algorithmic setting is appealing for its flexibility. The linear score formulation allows us to use rich features, while using ILP allows to easily incorporate structural constraints. Indeed, ILP has been proven valuable in various NLP tasks (Roth and Yih, 2007; Talukdar et al., 2012; Scaria et al., 2013). See Appendix A for our ILP formulation.

As a baseline, we experiment with an additional greedy inference algorithm, similar to the one described by Lapata (2003) for sentence ordering. The algorithm iteratively selects an outgoing edge (starting from the node $s$ ) that has the largest weight to a node that has not been visited so far, until all vertices are covered, at which point the path terminates by traveling to $f$.

\subsection{Learning}

The learning problem takes as input a dataset consisting of unordered sets of events, paired with a target ordering. We consider two types of learning algorithms for the edge-factored model in the previous section. The first learns in a global training setting using the averaged structured perceptron (Collins, 2002), with the decoding algorithm being either the one based on ILP (henceforth, GLOBAL-PRC), or the greedy one (GREEDY-PRC). Given a training instance $S$ and its correct label $h^{c}$, the structured perceptron calls the inference procedure as a subroutine and updates the weight vector $\theta$ according to the difference between the value of the feature function on the predicted path $\left(\sum_{h^{*}} \phi\left(h_{i}\right)\right)$ and on the correct path $\left(\sum_{h^{c}} \phi\left(h_{i}\right)\right)$.

The second learning algorithm we try is based on factored training. This algorithm maximizes the likelihood of a conditional log-linear model $p$ :

$$
\begin{gathered}
p\left(e_{2} \mid e_{1}, \theta, S\right)=\frac{\exp \left(\theta^{\top} \phi\left(e_{1}, e_{2}\right)\right)}{Z\left(\theta, S, e_{1}\right)} \\
Z\left(\theta, S, e_{1}\right)=\sum_{e:\left(e_{1}, e\right) \in E(S)} \exp \left(\theta^{\top} \phi\left(e_{1}, e\right)\right)
\end{gathered}
$$

where $e_{1}, e_{2} \in S \cup\{s, f\}$. This is a locally normalized log-linear model that gives the probability of transitioning to node $e_{2}$ from node $e_{1}$. Maximizing the score in Eq. 1 has an interpretation of finding the highest scoring path according to an edge-factored Markovian model, such that: 


$$
p(h \mid \theta, S)=\prod_{i=1}^{m+1} p\left(e_{i} \mid e_{i-1}, \theta, S\right),
$$

where $h=\left(h_{1}, \ldots, h_{m+1}\right)$ is a Hamiltonian path with $h_{i}=\left(e_{i-1}, e_{i}\right)$ being a directed edge in the path. Initial experimentation suggested that greedy inference (henceforth, GREEDY-LOGLIN) works better in practice than the ILP formulation for the locally-normalized model. We therefore do not report results on global inference with this log-linear model. We suspect that greedy inference works better with the log-linear model because it is trained locally, while the perceptron algorithm includes a global inference step in its training, and therefore better matches global decoding.

GREEDY-LOGLIN closely resembles the learning model of Lapata (2003), as both are firstorder Markovian and use the same (greedy) inference procedure. Lapata's model differs from GREEDY-LOGLIN in being a generative model, where each event is a tuple of features, and the transition probability between events is defined as the product of transition probabilities between feature pairs. GREEDY-LOGLIN is discriminative, so to be maximally comparable to the presented model.

\section{The Feature Set}

Table 1 presents the complete set of features. We consider three sets of features: Lexical encodes the written forms of the event pair predicates and objects; Brown uses Brown clusters (Brown et al., 1992) to encode similar information, but allows generalization between distributionally similar words; and Frequency encodes the empirical distribution of temporally-related phenomena.

The feature definitions make use of several functions. For brevity, we sometimes say that an event $e$ is $\left(a, c_{1}\right)$ if $e$ 's predicate is $a$ and its first argument is $c_{1}$, disregarding its other arguments. Let $\mathrm{C}$ be a reference corpus of recipes for collecting statistics. The function $B(w)$ gives the Brown cluster of a word $w$, as determined by clustering $\mathrm{C}$ into 50 clusters $\{1, \ldots, 50\}$. The function $\operatorname{ORD}(a, c)$ returns the mean ordinal number of an $(a, c)$ event in C. The ordinal number of the event $e_{i}$ in a recipe $\left(e_{1}, \ldots, e_{m}\right)$ is defined as $i-\frac{m}{2}$.

\begin{tabular}{|c|c|c|}
\hline & Template & Example \\
\hline \multirow{4}{*}{ 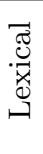 } & $\left(a^{1}, a^{2}\right)$ & (fry, add $)$ \\
\hline & $\overline{\left(a^{1}, c_{1}^{2}\right)}$ & $($ fry, oil $)$ \\
\hline & $\left(a^{2}, c_{1}^{1}\right)$ & (onions, add $)$ \\
\hline & $\left(c_{1}^{1}, c_{1}^{2}\right)$ & (onions, oil) \\
\hline \multirow{4}{*}{ 产 } & $\left(B\left(a^{1}\right), B\left(a^{2}\right)\right)$ & $(1,3)$ \\
\hline & $\begin{array}{l}\forall\left(k, k^{\prime}\right) \in K \\
\left|\left\{\left(c_{i}^{1}, c_{j}^{2}\right): B\left(c_{i}^{1}\right)=k, B\left(c_{j}^{2}\right)=k^{\prime}\right\}\right|\end{array}$ & $(5,4): 2$ \\
\hline & $\forall k \in K .\left|\left\{c_{i}^{2}: B\left(c_{i}^{2}\right)=k\right\}\right|$ & $12: 1$ \\
\hline & $\overline{B\left(a^{2}\right)}$ & 5 \\
\hline 3 & $\forall \ell \in L \cdot \log \left(\epsilon+P_{\ell}\left[\left(a^{2}, c_{1}^{2}\right) \mid\left(a^{1}, c_{1}^{1}\right)\right]\right)$ & -2.3 \\
\hline ప్ల & $\forall \ell \in L . \mathrm{PMI}_{\ell}\left(\left(a^{1}, c_{1}^{1}\right),\left(a^{2}, c_{1}^{2}\right)\right)$ & 3.1 \\
\hline 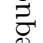 & $\forall \ell \in L . \mathrm{PMI}_{\ell}\left(\left(a^{2}, c_{1}^{2}\right),\left(a^{1}, c_{1}^{1}\right)\right)$ & -2.0 \\
\hline$=$ & $\operatorname{ORD}\left(a^{2}, c_{1}^{2}\right)$ & 3.2 \\
\hline
\end{tabular}

Table 1: Feature templates used for computing $\phi$. The templates operate on two events $\left\langle a^{1}, c_{1}^{1}, \ldots, c_{m_{1}}^{1}\right\rangle$ and $\left\langle a^{2}, c_{1}^{2}, \ldots, c_{m_{2}}^{2}\right\rangle$. $B(w)$ maps a word $w$ to its Brown cluster in $K=\{1, \ldots, 50\}$. $\mathrm{ORD}(e)$ returns the mean ordinal value of $e$. Feature templates which start with $\forall$ stand for multiple features, one for each element in the set quantified over. Non-numerical feature templates correspond to binary features. E.g., $($ fry,$a d d)$ as an instance of $\left(a^{1}, a^{2}\right)$ is a binary feature indicating the appearance of an event with $a^{1}=$ fry on one end of the edge and an event with $a^{2}=a d d$ on the other end of it. $\epsilon=10^{-3}$ in our experiments. See text for elaboration.

We further encode the tendency of two events to appear with temporal discourse connectives, such as "before" or "until." We define a linkage between two events as a triplet $\left(e_{1}, e_{2}, \ell\right) \in(U \times U \times L)$, where $L$ is the set of linkage types, defined according to their marker's written form. $\$ 6$ details the extraction process of linkages from recipes. We further include a special linkage type linear based on the order of events in the text, and consider every pair of events $e_{1}$ and $e_{2}$ that follow one another in a recipe as linked under this linkage type.

For each linkage type $\ell \in L$, we define an empirical probability distribution $P_{\ell}\left(\left(a, c_{1}\right),\left(a^{\prime}, c_{1}^{\prime}\right)\right)=$ $P\left(\left(a, c_{1}\right),\left(a^{\prime}, c_{1}^{\prime}\right) \mid \ell\right)$, based on simple counting. The function PMI gives the point-wise mutual information of two events and is defined as:

$$
\operatorname{PMI}_{\ell}\left((a, c),\left(a^{\prime}, c^{\prime}\right)\right)=\log \left(\frac{P_{\ell}\left(\left(a, c_{1}\right),\left(a^{\prime}, c_{1}^{\prime}\right)\right)}{P_{\ell}\left(a, c_{1}\right) \cdot P_{\ell}\left(a^{\prime}, c_{1}^{\prime}\right)}\right)
$$

Frequency-based features encode the empirical estimate of the probabilities that various pairs of features would occur one after the other or linked with a discourse marker. They are equivalent to using probabilities extracted from maximum likelihood estima- 
tion according to a bigram model in the discriminative learning. While some of this information is implicitly found in the lexical features, collecting frequency counts from a large training set is much quicker than running costly structured optimization. Rather the discriminative training can weigh the different empirical probabilities according to their discriminative power. Indeed we find that these features are important in practice and can result in high accuracy even after training on a small training set.

\section{The Recipe Dataset}

Data and Preprocessing. The data is extracted from a recipe repository found on the web. ${ }^{3}$ The recipes are given as free text. To extract event types we run the Stanford CoreNLP ${ }^{4}$ pipeline of a tokenizer, POS tagger, a lexical constituency parser (the englishPCFG parsing model) and extract typed Stanford dependencies (de Marneffe and Manning, 2008). As is common with web extractions, the recipes contain occasional spelling, grammatical and formatting errors. The corpus consists of 139 files, 73484 recipes, $1.02 \mathrm{M}$ events (13.8 events per recipe on average) and $11.05 \mathrm{M}$ words. ${ }^{5}$

Event Extraction. We focus on verbal events and do not extract nominal and adjectival argument structures, which are not as well supported by current parsing technology. Any verb is taken to define an event, aside from modal verbs, auxiliaries and secondary verbs. A secondary verb (e.g., "let," "begin") does not describe an action in its own right, but rather modifies an event introduced by another verb. We identify these verbs heuristically using a list given in Dixon (2005, p. 490-491) and a few simple rules defined over parse trees. E.g., from the sentence "you should begin to chop the onion," we extract a single event with a predicate "chop." Arguments are taken to be the immediate dependents of the predicate that have an argument dependency type (such as direct or indirect objects) according to the extracted Stanford dependencies. For prepositional phrases, we include the preposition as part of

\footnotetext{
$3_{\text {http: } / / \text { www.ffts.com/recipes.htm }}$

$4_{\text {http: } / / \text { nlp.stanford.edu/software/corenlp.shtml }}$

${ }^{5}$ Links to the original recipes, the preprocessed recipes and all extracted events can be found in http://homepages. inf.ed.ac.uk/oabend/event_order.html.
}

the argument. Argument indices are determined by their order in the text. The order of events is taken to be the order of their verbs in the text.

Linkage Extraction. We focus on a subset of linkage relations, which are relevant for temporal relations. We use Pitler and Nenkova's (2009) explicit discourse connectives classifier to identify temporal discourse linkers, discarding all other discourse linkers. Once a discourse linker has been detected, we heuristically extract its arguments (namely the pair of verbs it links) according to a deterministic extraction rule defined over the parse tree. We find 28 distinct connectives in our training set, where the 5 most common linkers "until," "then," "before," "when" and "as" cover over 95\% of the instances. We extract 36756 such linkages from the corpus, 0.5 linkages per recipe on average.

Temporal and Textual Ordering. In order to confirm that temporal and textual order of recipes are generally in agreement, we manually examine the first 20 recipes in our development set. One recipe was excluded as noise ${ }^{6}$, resulting in 19 recipes and 353 events. We identify the sources of misalignment between the linear order and the temporal order of the events. ${ }^{7} 13$ events $(3.7 \%)$ did not have any clear temporal orderings. These consisted of mostly negations and modalities (e.g., "do not overbrown!"), sub-section headings (e.g., "Preparation") or other general statements that do not constitute actions or states. For the remaining 340 events, we compare their linear and the temporal orderings.

We estimate the frequency of sub-sequences that contradict the temporal order and confirm that they occur only infrequently. We find that most disagreements fall into these two categories: (1) disjunctions between several events, only one of which will actually take place (e.g., "roll Springerle pin over dough, or press mold into top"); (2) a pair, or less commonly a triplet, of events are expressed in reverse order. For instance, "place on greased and floured cookie sheet," where greasing and flouring should occur before the placing action. We note that assuming the alignment of the temporal and textual order

\footnotetext{
${ }^{6}$ This did not result from an extraction problem, but rather from the recipe text itself being too noisy to interpret.

${ }^{7}$ Events are parsed manually so to avoid confounding the results with the parser's performance.
} 
of recipes does not suggest that the textual order is the only order of events that would yield the same outcome.

We compute the Kendall's Tau correlation, a standard measure for information ordering (Lapata, 2006), between the temporal and linear orderings for each recipe. In cases of several events that happen simultaneously (including disjunctions), we take their ordinals to be equal. For instance, for three events where the last two happen at the same time, we take their ordering to be $(1,2,2)$ in our analysis. We find that indeed temporal and textual orderings are in very high agreement, with 6 recipes of the 19 perfectly aligned. The average Kendall's Tau between the temporal ordering and the linear one is 0.924 .

\section{Experimental Setup}

Evaluation. We compute the accuracy of our algorithms by comparing the predicted order to the one in which the events are written. We first compute the number of exact matches, denoted with EXACT, namely the percentage of recipes in which the predicted and the textual orders are the same.

For a more detailed analysis of imperfect predictions, we compute the agreement between subsequences of the orderings. We borrow the notion of a "concordant pair" from the definition of Kendall's Tau and generalize it to capture agreement of longer sub-sequences. Two $k$-tuples of integers $\left(x_{1}, \ldots, x_{k}\right)$ and $\left(y_{1}, \ldots, y_{k}\right)$ are said to "agree in order" if for every $1 \leq i<j \leq k, x_{i}<x_{j}$ iff $y_{i}<y_{j}$. Given two orderings of the same recipe $O_{1}=\left(e_{\tau(1)}, \ldots, e_{\tau(m)}\right)$ and $O_{2}=\left(e_{\sigma(1)}, \ldots, e_{\sigma(m)}\right)$ (where $\tau$ and $\sigma$ are permutations over $[m]=\{1, \ldots, m\})$ and given a sequence of $k$ monotonically increasing indices $t=$ $\left(i_{1}, \ldots, i_{k}\right), t$ is said to be a "concordant $k$-tuple" of $O_{1}$ and $O_{2}$ if $\left(\tau\left(i_{1}\right), \ldots, \tau\left(i_{k}\right)\right)$ and $\left(\sigma\left(i_{1}\right), \ldots, \sigma\left(i_{k}\right)\right)$ agree in order, as defined above.

Denote the unordered recipes of the test data as $\left\{R_{i}\right\}_{i=1}^{N}$, where $R_{i}=\left\{e_{1}^{i}, \ldots, e_{m_{i}}^{i}\right\} \subset U$ for all $i$, and their target orderings $\Sigma=\left\{\sigma_{i}\right\}_{i=1}^{N}$, where $\sigma_{i}$ is a permutation over $\left[m_{i}\right]$. Assume we wish to evaluate a set of predicted orderings for this test data $\mathrm{T}=\left\{\tau_{i}\right\}_{i=1}^{N}$, where again $\tau_{i}$ is a permutation over $\left[m_{i}\right]$. Denote the number of concordant $k$-tuples of $\sigma_{i}$ and $\tau_{i}$ as $\operatorname{conc}\left(\sigma_{i}, \tau_{i}\right)$. The total number of of monotonically increasing $k$-tuples of indices is $\left(\begin{array}{c}m_{i} \\ k\end{array}\right)$. The $k$-wise (micro-averaged) accuracy of $\mathrm{T}$ with respect to $\Sigma$ is:

$$
\operatorname{acc}_{k}(\Sigma, \mathrm{T})=\frac{\sum_{i=1}^{N} \operatorname{conc}\left(\sigma_{i}, \tau_{i}\right)}{\sum_{i=1}^{N}\left(\begin{array}{c}
m_{i} \\
k
\end{array}\right)}
$$

Any $k$-tuples containing the start node $s$ or the end node $f$ are excluded, as their ordering is trivial. Recipes of length less than $k$ are discarded when computing $\operatorname{acc}_{k}$. A micro-averaged accuracy measure is used so as not to disproportionately weigh short recipes. However, in order to allow comparison to mean Kendall's Tau, commonly used in works on order learning, we further report a macroaveraged $\mathrm{acc}_{2}$ by computing $\mathrm{acc}_{2}$ for each recipe separately, and taking the average of resulting accuracy levels. Average Kendall's Tau can now be computed by $2 \mathrm{acc}_{2}-1$ for the macro-averaged $\mathrm{acc}_{2}$ score.

Data. We randomly partition the text into training, test and development sets, taking an 80-10-10 percent split. We do not partition the individual files so as to avoid statistical artifacts introduced by recipe duplications or near-duplications. The training, development and test sets contain 58038, 7667 and 7779 recipes respectively. The total number of feature template instantiations in the training data is $8.94 \mathrm{M}$.

Baselines and Algorithms. We compare three learning algorithms. GLOBAL-PRC is the structured perceptron algorithm that uses ILP inference. GREEDY-PRC is a structured perceptron in which inference is done greedily. GREEDY-LOGLIN is the locally normalized log-linear model with greedy inference. RANDOM randomly (uniformly) selects a permutation of the recipe's events.

Experimental Settings. The structured perceptron algorithms, GLOBAL-PRC and GREEDY-PRC, are run with a learning rate of 0.1 for 3 iterations. To avoid exceedingly long runs, we set a time limit in seconds $\beta$ on the running time of each ILP inference stage used in GLOBAL-PRC. We consider two training scenarios: $4 K$, which trains on the first $4 \mathrm{~K}$ recipes of the training set, and $58 K$, which trains on the full training data of $58 \mathrm{~K}$ recipes. In GLOBAL-PRC we set $\beta$ to be 30 seconds for the $4 \mathrm{~K}$ 


\begin{tabular}{|c|c|c|c|c|c|c|}
\hline \multirow{4}{*}{ Alg. } & \multicolumn{2}{|c|}{$\mathrm{acc}_{2}$} & $\mathrm{acc}_{3}$ & $\mathrm{acc}_{4}$ & EXACT \\
\cline { 2 - 7 } & MI & MA & & & \\
\hline & GLOBAL-PRC & $\mathbf{7 1 . 2}$ & $\mathbf{7 7 . 7}$ & $\mathbf{4 4 . 7}$ & $\mathbf{2 7 . 9}$ & $\mathbf{3 5 . 1}$ \\
\hline & GREEDY-PRC & 60.8 & 68.0 & 30.6 & 15.0 & 20.4 \\
\hline & GREEDY-LOGLIN & 65.6 & 71.5 & 35.8 & 18.7 & 21.0 \\
\hline \multirow{2}{*}{$\backsim$} & GLOBAL-PRC & 68.9 & 76.4 & 41.3 & 24.8 & 34.4 \\
\hline \multirow{2}{*}{} & GREEDY-PRC & 60.7 & 67.8 & 30.6 & 15.2 & 20.5 \\
\cline { 2 - 7 } & GREEDY-LOGLIN & 66.3 & 72.4 & 36.6 & 19.4 & 21.3 \\
\hline & RANDOM & 50.0 & 51.2 & 16.7 & 4.2 & 0.5 \\
\hline
\end{tabular}

Table 2: Accuracy of the different models on the test data in percents. Columns correspond to evaluation measures, namely accuracy of sub-sequences of lengths 2 (micro and macro averages), 3 and 4, and exact match. The upper (lower) part presents results for a training set of $4 \mathrm{~K}(58 \mathrm{~K})$ samples. GLOBAL-PRC is run with $\beta=30$ for $4 \mathrm{~K}$ and with $\beta=5$ for $58 \mathrm{~K}$. In $58 \mathrm{~K}$, all models are run with the Full feature set. In $4 \mathrm{~K}$, following prior experimentation on the development set, we select the best performing feature sets (Full for GREEDY-LOGLIN and GREEDY-PRC; Fr + Lex for GLOBAL-PRC).

scenario, and 5 seconds in the $58 \mathrm{~K}$ scenario. The number of threads was limited to 3 . Where the time limit is reached before an optimal solution is found, the highest scoring Hamiltonian path found up to that point is returned by the ILP solver. In the infrequent samples where no feasible solution is found during training, the sample is skipped over, while at test time, we perform greedy inference instead.

We define the following feature sets. Fr includes only features of class Frequency, while Fr + Lex includes features from both the Frequency and Lexical categories. Full includes all feature sets. All above feature sets take $\mathrm{C}$, the reference corpus for computing FREQUENCY features, to be the entire $58 \mathrm{~K}$ training samples in both scenarios. In the 4K scenario, we also experiment with FrLim, which includes all features, but takes $\mathrm{C}$ to contain only the $4 \mathrm{~K}$ samples of the training data.

We use the Gurobi package for ILP. ${ }^{8}$ Brown clusters are extracted from the $58 \mathrm{~K}$ samples of the training data using Liang's implementation. ${ }^{9}$ The convex log-likelihood function of GREEDY-LOGLIN is optimized using LBFGS. All features are selected and all parameters are tuned using the development set.

\section{Results}

Table 2 presents the results of the three major algorithms in the two main scenarios $58 K$ and $4 K$.

\footnotetext{
$8_{\text {http: //www.gurobi.com }}$

9 https://github.com/percyliang/brown-cluster
}

\begin{tabular}{|c|c|c|c|c|c|c|}
\hline$\beta$ & Set & \multicolumn{2}{|c|}{$\mathrm{acc}_{2}$} & $\mathrm{acc}_{3}$ & $\mathrm{acc}_{4}$ & EXACT \\
\hline \hline 30 & & MI & MA & & & \\
\cline { 2 - 7 } & FrLim & 55.9 & 61.5 & 21.6 & 6.9 & 8.2 \\
\cline { 2 - 7 } & Fr & 68.7 & 75.9 & 40.6 & $\mathbf{2 3 . 9}$ & 31.7 \\
\cline { 2 - 7 } & Fr + Le & $\mathbf{6 8 . 9}$ & $\mathbf{7 6 . 2}$ & $\mathbf{4 0 . 7}$ & 23.8 & $\mathbf{3 2 . 1}$ \\
\cline { 2 - 7 } & Full & 68.4 & 76.0 & 39.9 & 23.1 & 31.8 \\
\hline 5 & FrLim & 55.1 & 60.9 & 20.9 & 6.5 & 8.2 \\
\cline { 2 - 7 } & Fr & 65.9 & 74.2 & 36.0 & 19.3 & 30.4 \\
\cline { 2 - 7 } & Fr + Le & 66.2 & 74.3 & 36.8 & 20.4 & 30.7 \\
\cline { 2 - 7 } & Full & 66.3 & 74.5 & 36.9 & 20.4 & 30.4 \\
\hline
\end{tabular}

Table 3: The performance of GLOBAL-PRC on the development set in various settings ( $4 \mathrm{~K}$ scenario). Columns correspond to evaluation measures, namely accuracy of sub-sequences of lengths 2 (micro and macro averages), 3 and 4, and exact match. The upper (lower) part of the table presents results for a time limit of $\beta=30$ (5). Fr includes the Frequency features estimated on $58 \mathrm{~K}$ recipes. Fr + Le further includes Lexical features. Full includes all features. FrLim includes all features, where Frequency features are estimated only on $4 \mathrm{~K}$ recipes.

We find that the structured perceptron algorithm, GLOBAL-PRC, obtains the best results in both cases and under all evaluation measures. The importance of global optimization was also stressed in other works on event ordering (Chambers and Jurafsky, 2008a; Talukdar et al., 2012).

In order to assess the contribution of the different components of the model of the best scoring model, GLOBAL-PRC, we compare the performance of the different feature sets and settings of $\beta$ on the development set in $4 \mathrm{~K}$ (Table 3 ). Results reveal the strong impact of the Frequency feature set on the results. Using this category set alone (Fr) yields slightly lower results than using the full feature set, while estimating the Frequency features on a small corpus (FrLim) lowers results dramatically. Adding Lexical and Brown features yields a small improvement over using Frequency alone.

While Table 3 demonstrates the importance of $\beta$ in the performance of GLOBAL-PRC, it also shows that on a limited time budget, a small training set and few features (4K, Fr) and a reasonably small $\beta$ (5) can yield competitive results. Increasing $\beta$ from 5 to 30 generally improves results by 2 to 3 percent absolute. The importance of $\beta$ is further demonstrated in Table 2, where performance with $4 \mathrm{~K}$ training instances and $\beta=30$ is better than with $58 \mathrm{~K}$ training instances and $\beta=5$. Preliminary experiments conducted on the development data with higher values of $\beta$ of 60 and 120 suggest that further increasing $\beta$ 
yields no further improvement.

Previous studies evaluated their models on the related problem of distinguishing randomly permuted and correctly ordered chains of events (\$2). In this paper we generalize this task to complete event ordering. In order to demonstrate the relative difficulty of the tasks, we apply our highest scoring model $(4 \mathrm{~K}, \mathrm{Fr}+\mathrm{Le})$ to the binary task (without re-training it). We do so by computing the percentage of cases in which the correct ordering obtains a higher score than an average ordering. The high resulting accuracy of $93 \%$, as opposed to considerably lower accuracies obtained under ordering evaluation measures, reflects the relative difficulty of the tasks.

The proposed edge-factored model can easily capture pair-wise ordering relations between events, but is more limited in accounting for relations between larger sets of events. A simple way of doing so is by adding the feature $\sum_{e} P\left(e_{i} \mid e\right) P\left(e \mid e_{j}\right)$ between events $e_{i}$ and $e_{j}$ (in addition to the regular transition probabilities $\left.P\left(e_{i} \mid e_{j}\right)\right)$. However, preliminary experimentation with this technique did not yield improved performance. Future work will address higher-order models that straightforwardly account for such long-distance dependencies.

To qualitatively assess what generalizations are learned by the model, we apply GLOBAL-PRC to the development data and look at what event pairs obtained either particularly high or particularly low results. For each pair of predicates and their first arguments $\left(a^{1}, c_{1}^{1}\right),\left(a^{2}, c_{1}^{2}\right)$, we compute the average weight of an edge connecting events of these types, discarding pairs of frequency less than 20 .

The 20 highest scoring edges contain pairs such as ("add," "mixing after addition"), ("beat whites," "fold into mixture") and ("cover for minutes," "cook"), in addition to a few noisy pairs resulting from parser errors. The 20 lowest scoring edges contain event pairs that are likely to appear in the opposite order. 11 of the cases include as a first argument the predicates "serve," "cool" or "chill," which are likely to occur at the end of a recipe. 3 other edges linked duplications (e.g., ("reduce heat," "reduce heat")), which are indeed unlikely to immediately follow one another. These findings suggest the importance of detecting both lexical pairs that are unlikely to follow one another, in addition to those that are likely to.

\section{Conclusion}

We addressed the problem of lexical event ordering, and developed an edge-factored model for tackling it. We rely on temporally aligned texts, using a new dataset of cooking recipes as a test case, thereby avoiding the need for costly and error-prone manual annotation. We present results of a pair-wise accuracy of over $70 \%$ using a basic set of features, and show the utility of the structured perceptron algorithm over simpler greedy and local approaches. The setup we explore, which uses a discriminative model and an ILP formulation, is easy to extend both in terms of features and in terms of more complex formal constraints and edge dependencies, as was done in graph-based dependency parsing (McDonald et al., 2005). Future work will address the extension of the feature set and model, and the application of this model to temporal semantics and planning tasks. We will further address the application of semi-supervised variants of the proposed techniques (e.g., self-training) to other domains, where no sizable corpora of temporally aligned data can be found.

\section{Acknowledgments}

We would like to thank Nathan Schneider, Roy Schwartz, Bonnie Webber and the members of the Probmodels group at the University of Edinburgh for helpful comments. This work was supported by ERC Advanced Fellowship 249520 GRAMPLUS.

\section{Appendix A: Maximal Hamiltonian Path}

Let $G(S)=(S \cup\{s, f\}, E(S))$ be an almostcomplete directed graph with $E=E(S)=(S \cup$ $\{s\}) \times(S \cup\{f\})$. Let $c_{i j} \in \mathbb{R}$ be weights for its edges $((i, j) \in E)$. A Hamiltonian path between $s, f \in V$ can be found by solving the following program, returning $P=\left\{(i, j) \mid x_{i j}=1\right\}$.

$$
\begin{gathered}
\max _{\substack{x_{i j} \in\{0,1\}:(i, j) \in E \\
u_{i} \in \mathbb{Z}: i \in V}} \sum_{i \neq j}^{n} c_{i j} x_{i j} \\
\text { s.t. } \sum_{\substack{i=0, i \neq j \\
u_{i}-u_{j}+|V| x_{i j} \leq|V|-1 \quad \forall(i, j) \in E}} x_{i j}=1 \forall j \neq s ; \quad \sum_{j=0, j \neq i}^{n} x_{i j}=1 \quad \forall i \neq e ;
\end{gathered}
$$




\section{References}

Niranjan Balasubramanian, Stephen Soderland, Mausam, and Oren Etzioni. 2013. Generating coherent event schemas at scale. In EMNLP' 13, pages 1721-1731.

Regina Barzilay and Mirella Lapata. 2008. Modeling local coherence: An entity-based approach. Computational Linguistics, 34(1):1-34.

Mario Bollini, Stefanie Tellex, Tyler Thompson, Nicholas Roy, and Daniela Rus. 2013. Interpreting and executing recipes with a cooking robot. In Experimental Robotics, pages 481-495. Springer.

Peter F Brown, Peter V Desouza, Robert L Mercer, Vincent J Della Pietra, and Jenifer C Lai. 1992. Classbased n-gram models of natural language. Computational Linguistics, 18(4):467-479.

Taylor Cassidy, Bill McDowell, Nathanael Chambers, and Steven Bethard. 2014. An annotation framework for dense event ordering. In ACL '14: Short Papers, pages 501-506.

Nathanael Chambers and Dan Jurafsky. 2008a. Jointly combining implicit constraints improves temporal ordering. In EMNLP '08, pages 698-706.

Nathanael Chambers and Dan Jurafsky. 2008b. Unsupervised learning of narrative event chains. In $A C L-H L T$ '08, pages 789-797.

Nathanael Chambers and Dan Jurafsky. 2009. Unsupervised learning of narrative schemas and their participants. In ACL-IJCNLP '09, pages 602-610, Suntec, Singapore, August.

Timothy Chklovski and Patrick Pantel. 2004. Verbocean: Mining the web for fine-grained semantic verb relations. In EMNLP' 04 , pages 33-40.

Philipp Cimiano, Janna Lüker, David Nagel, and Christina Unger. 2013. Exploiting ontology lexica for generating natural language texts from RDF data. In European Workshop on Natural Language Generation, pages 10-19.

Michael Collins. 2002. Discriminative training methods for hidden markov models: Theory and experiments with perceptron algorithms. In EMNLP '02, pages 18.

Ido Dagan, Dan Roth, Mark Sammons, and Fabio Massimo Zanzotto. 2013. Recognizing textual entailment: Models and applications. Synthesis Lectures on $\mathrm{Hu}$ man Language Technologies, 6(4):1-220.

Dmitry Davidov, Ari Rappoport, and Moshe Koppel. 2007. Fully unsupervised discovery of conceptspecific relationships by web mining. In $A C L$ '07, pages 232-239.

Marie-Catherine de Marneffe and Christopher D Manning. 2008. The Stanford typed dependencies representation. In the COLING'O8 workshop on CrossFramework and Cross-Domain Parser Evaluation, pages $1-8$.
Robert M.W. Dixon. 2005. A Semantic Approach To English Grammar. Oxford University Press.

Jennifer D'Souza and Vincent Ng. 2013. Classifying temporal relations with rich linguistic knowledge. In NAACL-HLT'13, pages 918-927.

Lea Frermann, Ivan Titov, and Manfred Pinkal. 2014. A hierarchical bayesian model for unsupervised induction of script knowledge. In EACL'14, pages 49-57.

Bram Jans, Steven Bethard, Ivan Vulić, and MarieFrancine Moens. 2012. Skip n-grams and ranking functions for predicting script events. In EACL ' 12 , pages 336-344.

Maria Lapata and Alex Lascarides. 2006. Learning sentence-internal temporal relations. Journal of Artificial Intelligence Research (JAIR), 27:85-117.

Mirella Lapata. 2003. Probabilistic text structuring: Experiments with sentence ordering. In $A C L$ '03, pages 545-552.

Mirella Lapata. 2006. Automatic evaluation of information ordering: Kendall's tau. Computational Linguistics, 32(4):471-484.

Xiao Ling and Daniel S Weld. 2010. Temporal information extraction. In $A A A I$ ' 10 , pages 1385 - 1390.

Jonathan Malmaud, Earl Wagner, Nancy Chang, and Kevin Murphy. 2014. Cooking with semantics. In the ACL 2014 Workshop on Semantic Parsing, pages 33-38.

Inderjeet Mani, Marc Verhagen, Ben Wellner, Chong Min Lee, and James Pustejovsky. 2006. Machine learning of temporal relations. In $A C L-C O L I N G{ }^{\prime} 06$, pages 753-760.

Mehdi Manshadi, Reid Swanson, and Andrew S. Gordon. 2008. Learning a probabilistic model of event sequences from internet weblog stories. In FLAIRS '08, pages 159-164.

Ryan McDonald, Koby Crammer, and Fernando Pereira. 2005. Online large-margin training of dependency parsers. In $A C L$ '05, pages 91-98.

Paramita Mirza and Sara Tonelli. 2014. Classifying temporal relations with simple features. In EACL '14, pages 308-317, Gothenburg, Sweden, April.

Ashutosh Modi and Ivan Titov. 2014. Inducing neural models of script knowledge. In CoNLL'14, pages 4957.

Kira Mourão, Luke Zettlemoyer, Ronald Petrick, and Mark Steedman. 2012. Learning STRIPS operators from noisy and incomplete observations. In UAI' 12 .

Karl Pichotta and Raymond Mooney. 2014. Statistical script learning with multi-argument events. In $E A C L$ '14, pages 220-229.

Emily Pitler and Ani Nenkova. 2009. Using syntax to disambiguate explicit discourse connectives in text. In ACL-IJCNLP '09: Short Papers, pages 13-16. 
James Pustejovsky, José M Castano, Robert Ingria, Robert J Gaizauskas, Andrea Setzer, Graham Katz, and Dragomir R Radev. 2003. TimeML: Robust specification of event and temporal expressions in text. New directions in question answering, 3:28-34.

Michaela Regneri, Alexander Koller, and Manfred Pinkal. 2010. Learning script knowledge with web experiments. In $A C L$ '10, pages 979-988.

Michaela Regneri, Marcus Rohrbach, Dominikus Wetzel, Stefan Thater, Bernt Schiele, and Manfred Pinkal. 2013. Grounding action descriptions in videos. Transactions of the Association for Computational Linguistics (TACL), 1:25-36.

Ehud Reiter, Robert Dale, and Zhiwei Feng. 2000. Building natural language generation systems, volume 33. Cambridge: Cambridge university press.

Dan Roth and Wen-tau Yih. 2007. Global inference for entity and relation identification via a linear programming formulation. In Lise Getoor and Ben Taskar, editors, Introduction to Statistical Relational Learning. MIT Press.

Aju Thalappillil Scaria, Jonathan Berant, Mengqiu Wang, Christopher D Manning, Justin Lewis, Brittany Harding, and Peter Clark. 2013. Learning biological processes with global constraints. In EMNLP' 13 .

Mark Steedman. 2002. Plans, affordances, and combinatory grammar. Linguistics and Philosophy, 25(56):723-753.

Partha Pratim Talukdar, Derry Wijaya, and Tom Mitchell. 2012. Acquiring temporal constraints between relations. In CIKM'12, pages 992-1001.

Dan Tasse and Noah A Smith. 2008. SOUR CREAM: Toward semantic processing of recipes. Technical report, Technical Report CMU-LTI-08-005, Carnegie Mellon University, Pittsburgh, PA.

Roy Tromble and Jason Eisner. 2009. Learning linear ordering problems for better translation. In EMNLP '09, pages 1007-1016.

Naushad UzZaman, Hector Llorens, Leon Derczynski, James Allen, Marc Verhagen, and James Pustejovsky. 2013. Semeval-2013 task 1: Tempeval-3: Evaluating time expressions, events, and temporal relations. In *SEM-SemEval'13, pages 1-9.

Katsumasa Yoshikawa, Sebastian Riedel, Masayuki Asahara, and Yuji Matsumoto. 2009. Jointly identifying temporal relations with markov logic. In ACL-IJCNLP '09, pages 405-413. 